\title{
DE L'EFFICACITE DES MESURES DE CONVERGENCE POUR PREPARER LE PASSAGE AUX IAS/IFRS EN FRANCE
}

\author{
Salma DAMAK-AYADI \\ CREFIGE, Université de Paris Dauphine \\ UREMO, IHEC Carthage \\ salmadamak@yahoo.fr
}

\begin{abstract}
Résumé
Ce papier a pour objet d'étudier la convergence du droit comptable français vers les IAS/IFRS et la comparabilité des résultats obtenus selon les deux référentiels. Tout d'abord, nous avons essayé d'exposer les différentes stratégies de normalisation en se basant sur deux critères relatifs au degré d'intervention de l'Etat et des organismes professionnels du pays et au recours aux IAS/IFRS. Nous avons pu distinguer quatre stratégies possibles ; une stratégie de délégation de la normalisation à l'IASB, une stratégie de convergence vers les IAS/IFRS, une stratégie de délégation de la normalisation aux autres organismes de normalisation internationaux et une stratégie d'auto-normalisation. Ensuite, nous avons essayé d'approfondir l'analyse de la stratégie de convergence vers les IAS/IFRS à travers l'étude du cas de la France et plus particulièrement de l'efficacité des mesures de convergence entreprises en France afin de préparer le passage aux IAS/IFRS en 2005. L'étude concerne un échantillon de 30 entreprises françaises cotées en bourse ayant publié leurs états financiers selon les référentiels national et international en 2004 et 2005. Les résultats montrent que la comparabilité des résultats obtenus reste limitée surtout pour les petites entreprises.

Mots clés : Convergence comptable, comparabilité, IASB, IAS/IFRS, entreprises françaises

Abstract:

This research investigates the comparability and convergence of the French standards and the IFRS in 2004 and 2005. First, this paper exposes different strategies about accounting standardisation in presence of the IASB's standards. Based on two criteria's; the degree of implication of public authorities and national professional groups and the degree of adoption of IAS/IFRS, we distinguished four strategies. Second, the investigation of comparability and convergence of two sets of accounting standards involves a sample of 30 French companies which reporting under French standards and IAS/IFRS. The results show a lack of comparability.

Key words: Accounting convergence, comparability, IASB, IAS/IFRS, French firms
\end{abstract}




\section{INTRODUCTION}

La normalisation comptable a connu d'énormes mutations depuis le $20^{\text {ème }}$ siècle. L'intervention de la réglementation, le développement des organismes professionnels, l'internationalisation des marchés, l'importance accrue accordée aux informations financières ont amené les acteurs sociaux de la normalisation (qu'ils soient producteurs, utilisateurs, auditeurs ou normalisateurs de l'information comptable) à être plus attentifs à ce qui se passe à l'échelle internationale. La normalisation nationale, qui était la plus active depuis quelques années, a cédé la place à une normalisation internationale émergente qui vise la standardisation. Ces dernières années, l'IASB a renforcé sa position de leader en matière de normalisation comptable avec l'adoption à partir de 2005 des IAS/IFRS ${ }^{1}$ pour la préparation des comptes consolidés des groupes d'entreprises européennes cotées en bourse.

Tout d'abord, nous avons essayé de répondre à une question fondamentale qui se pose en ce moment dans certains pays en matière de normalisation comptable : quelle stratégie de normalisation faut-il adopter face à l'IASB ?

En se basant sur deux critères relatifs au degré d'intervention de l'Etat et des organismes professionnels du pays à la mormalisation comptable et au recours aux normes internationales de l'IASB, nous avons pu distinguer quatre stratégies possibles; une stratégie de délégation de la normalisation à l'IASB, une stratégie de convergence vers les IAS/IFRS, une stratégie de délégation de la normalisation aux autres organismes de normalisation internationaux et une stratégie d'auto-normalisation. Ensuite, nous avons essayé d'approfondir la stratégie de convergence vers les IAS/IFRS à travers l'étude du cas de la France, et plus particulièrement, nous avons traité de l'efficacité des mesures de convergence entreprises ces dernières années afin de préparer le passage aux IAS/IFRS en 2005.

\footnotetext{
${ }^{1}$ le référentiel IAS/IFRS représente les normes comptables et les normes d'interprétations adoptées par l'IASB. Il correspond :

- Aux normes comptables internationales IAS (International Accounting Standard) : elles étaient 31 au 31 mars 2004 numérotées de 1 à 41 (les normes IAS 3, 4, 5, 6, 9, 13, 15, 22, 25 et 35 ayant été remplacées ou supprimées).

- Aux normes internationales d'information financière IFRS (International Financial Reporting Standard) : elles étaient 5 au 31 mars 2004 numérotées de 1 à 5. Deux nouvelles normes ont été adoptées depuis le 31 mars 2004 (IFRS 6 qui entre en vigueur pour les exercices ouverts à compter du $1^{\text {er }}$ janvier 2006 et IFRS 7 à compter du $1^{\text {er }}$ janvier 2007)

- Aux normes d'interprétations se rapportant à ces normes comptables SIC (Standing Interpretations Committee) 11 au 31 mars 2004 et IFRIC 7 au 31 décembre 2005.
} 
L'analyse a porté sur la comparabilité des informations comptables publiées selon les référentiels français et international et de leur relation avec les caractéristiques des entreprises. Ainsi, ce papier comporte quatre parties. Dans la première, nous avons défini les concepts clés liés à la notion de convergence comptable, dans la deuxième, nous avons proposé une typologie de stratégies de normalisation comptables possibles en présence de l'IASB, dans la troisième, nous avons procédé à une revue de la littérature des principaux travaux réalisés à l'échelle internationale traitant de l'harmonisation comptable internationale et dans la dernière, nous avons présenté les résultats de notre étude.

\section{EST-IL QUESTION DE NORMALISATION, D'HARMONISATION OU DE CONVERGENCE COMPTABLE INTERNATIONALE ?}

\subsection{Définition des concepts}

Lorsqu'on veut parler de la normalisation comptable, plusieurs concepts surgissent : la régulation, la réglementation, la standardisation, l'harmonisation, la convergence comptable...

Pour définir la normalisation comptable, Bernard Colasse (2004a, p. 1) revient à la notion de régulation qu'il définit comme étant «le processus de production, de mise en ouvre et de contrôle de l'application des normes comptables dans un espace géographique donné ». Selon cet auteur, la notion de normalisation ne fait référence qu'à l'aspect de la production des normes. D'autres considèrent que la normalisation est la traduction francophone de la «standardisation», mais elle ne coïncide pas forcément avec elle puisque cette dernière permet d'atteindre une uniformité totale, elle a conduit à l'adoption d'une seule règle comptable dont l'application sera universelle (Garrido et al. 2002). Cette standardisation n'est possible qu'au travers d'une harmonisation comptable internationale. L'une des premières définitions de l'harmonisation comptable était celle de Nobes et Parker (1981, p. 329), «il s'agit d'un processus destiné à accroître la compatibilité des pratiques comptables grâce à une limitation de leur niveau de variabilité ». Selon Colasse (2000, p.787), «l'harmonisation comptable est un processus institutionnel, ayant pour objet de mettre en convergence les normes et les pratiques comptables nationales et par conséquent, de faciliter la comparaison des états comptables produits par des entreprises de pays différents ». Selon Choi et al. (2001), l'harmonisation comptable a trois facettes, l'harmonisation des normes comptables, 
l'harmonisation de la diffusion d'informations comptables et l'harmonisation des normes d'audit. Dans les études antérieures, les auteurs (Canibano et Mora 2000, Larson et Kenny 2000, Haverty 2006, Garrido et al. 2002) ont fait la distinction entre l'harmonisation formelle ${ }^{2}$ et informelle ${ }^{3}$. L'harmonisation formelle se réfère à l'harmonisation des réglementations alors que l'harmonisation informelle se réfère à l'harmonisation des pratiques.

Mais cette notion d'harmonisation est parfois associée à celle de la convergence en relation avec le travail fait par l'IASB. En effet, Hussey et Ong (2005, p.229) la définissent comme «le processus mené par l'IASB pour éliminer les différences actuelles qui existent avec les normes comptables nationales et éviter les futures différences afin d'atteindre une harmonisation comptable internationale ». Le paragraphe suivant retrace la contribution de l'IASB à l'harmonisation comptable internationale surtout au niveau européen.

\subsection{Les tentatives d'harmonisation comptable}

\subsubsection{Au niveau européen}

En Europe, l'harmonisation comptable a commencé dans les années 1970 et s'est faite au moyen des directives que les Etats membres ont transposé progressivement dans leurs législations nationales. Il ne s'agissait pas d'imposer des règles communes aux entreprises européennes mais plus simplement de définir un cadre qui limitât les écarts entre les Etats membres. Cette ambition s'inscrivait dans un programme plus général d'harmonisation des lois sur les sociétés, visant à coordonner les garanties exigées pour protéger les intérêts des associés et des tiers. La $4^{\text {ème }}$ directive, du 25 juillet 1978, traite des objectifs de la présentation et du contenu des comptes annuels des sociétés de capitaux et la ł̇̀me directive, du 13 juin 1983, traite des comptes des groupes. Si Colasse (2002) parle de mésaventures de cette tentative européenne d'harmonisation, plusieurs autres chercheurs (Chiapello 2005) la considèrent comme un véritable échec à cause de la lenteur dans l'application de ces directives et la diversité des options offertes. De plus, certaines questions comptables ont évolué beaucoup plus vite par rapport au temps politique nécessaire pour travailler avec l'outil juridique des directives. En 1995, l’Union Européenne a changé de stratégie et s'est tournée vers la normalisation internationale. En juin 2000, la CE annonce l'intention de rendre obligatoire les normes de l'IASB pour les sociétés européennes cotées. Le projet du règlement européen fût rédigé en février 2001 et approuvé par le parlement européen (CE n¹606/2002)

\footnotetext{
${ }^{2}$ De jure

${ }^{3}$ De facto
} 
en mars 2002. Ce règlement a nécessité la création d'un organisme d'approbation appelé Comité de Réglementation Comptable Européen, composé de représentants des différents Etats membres de l'Union Européenne qui donne son avis sur l'application ou le rejet des IAS/IFRS. Ce comité se fait assister par l'European Financial Reporting Advisory Group créé en 2001 qui a pour mission de fournir l'expertise technique dont le Comité de Réglementation Comptable Européen a besoin pour agréer les normes de l'IASB. Actuellement, la majorité des normes internationales ont été approuvées par la CE. Si l'harmonisation comptable par les directives n'a pas attiré beaucoup d'attention, la décision d'adoption des IAS/IFRS en Europe a fait couler beaucoup d'ancre ${ }^{4}$.

\subsubsection{Au niveau international : L'IASB}

L'IASB a été créé sous le nom de l'IASC à Londres en 1973 par les représentants des principales organisations comptables de neuf pays ${ }^{5}$. Son fonctionnement actuel a été totalement repensé en 2001 autour de différents conseils et assemblées qui ont pour missions d'établir, de publier, de vérifier et de faire accepter une règle comptable au plan international. L'ensemble IASB est maintenant formé de quatre organismes :

- l'IASB qui est l'organe central de l'organisation,

- l'International Accounting Standard Committee Foundation, une fondation chargée de superviser l'organisation et de développer et de faire appliquer les normes comptables internationales,

- l'International Financial Reporting Interpretation Committee (IFRIC), un comité chargé de répondre aux problèmes d'interprétation,

- le Standards Advisory Council (SAC) un comité consultatif de normalisation.

Les objectifs de l'IASB étaient de formuler et de publier dans l'intérêt général les normes comptables internationales, de promouvoir leur application dans le monde et de travailler de façon générale à l'amélioration et à l'harmonisation des réglementations, des normes comptables et des procédures relatives à la présentation des comptes.

Au début, les normes de l'IASB n'engageaient que ses membres qui, de toute façon, devraient respecter la réglementation de leurs pays, et elles ne étaient pas obligatoires pour les entreprises. Néanmoins, elles n'étaient pas sans influence sur les pratiques et réglementations

\footnotetext{
${ }^{4}$ Delesalle 2002, Colasse 2004a, Heem et Aonzo 2003, Chiapello 2005, Amblard 2004, Blic 2004, Colasse 2004b, Haller 2002, Van Hulle 1996, Larson et Street 2004, Achleitner 1995, Alexander et Schwencke 2003, Canibano et Mora 2000, Street et Larson 2004, Jermakowicz et Gornick-Tomaszewski 2006, Delvaille et al. 2005, Dumontier et Raffournier 1998, Haller et Eierle 2004, Schipper 2005, Murphy 2000, Street et Gray 2002, Tarca 2004

${ }^{5}$ Allemagne, France, Pays-Bas, Royaume Unis, Australie, Etats Unis, Mexique, Canada et l'Irlande
} 
nationales. L'OICV a récemment recommandé à ses membres l'utilisation des IAS/IFRS. Selon un rapport publié sur le site de l'IASB, 32 pays ont adopté les normes internationales en 2003. Ce chiffre a augmenté ces dernières années surtout après l'application du règlement européen ${ }^{6}$. Même les normalisateurs aux Etats Unis, qui avaient montré auparavant une politique plutôt hostile vis-à-vis des normalisations étrangères ${ }^{7}$, sont entrés depuis 2002 dans un projet commun de révision du cadre conceptuel de la comptabilité avec l'IASB ${ }^{8}$.

Face à ce nouveau mouvement d'harmonisation internationale mené par l'IASB, quelle stratégie faut-il adopter? un alignement ou une adaptation des IAS/IFRS ? quel rôle joué par les organismes de normalisation nationaux dans ces cas?

Certains éléments de réponse seront fournis dans le paragraphe suivant.

\section{LES STRATEGIES DE NORMALISATION COMPTABLE}

\subsection{Proposition d'une typologie}

Chaque pays possède son propre système de normalisation et de réglementation comptable. Ce dernier dépend essentiellement du rôle joué par l'Etat dans la production et la mise en application des normes comptables mais pourrait également être influencé par des facteurs de contingence (système économique, cadre juridique, relation entre comptabilité et fiscalité).

Dans la littérature, la normalisation comptable a été souvent rattachée à la nature des personnes ou des organismes participant à cette normalisation, c'est ainsi qu'on distingue entre:

- la normalisation nationale et la normalisation internationale (Collette et Richard 2000)

- la normalisation par le marché libre, la normalisation publique, la normalisation privée et la normalisation mixte (Belkaoui 1984)

${ }^{6}$ Source : site web Deloitte « IAS plus » en 2007
\begin{tabular}{|l|l|l|l|l|}
\hline & Entreprises & cotées & \multicolumn{1}{l|}{ Entreprises } & non cotées \\
\hline Normes IAS/IFRS & Obligatoires & Permises & Obligatoires & Permises \\
\hline Nombre de pays & 82 & 23 & 38 & 36 \\
\hline
\end{tabular}

\footnotetext{
${ }^{7}$ Même celle de l'IASB, la SEC a réservé son approbation à la résolution de l'OICV recommandant à tous ses membres d'accepter l'utilisation des normes IFRS par les sociétés étrangères en 2000. Elle n'accepte que les normes américaines et exige un document de retraitement et de réconciliation pour les états financiers présentés autrement.

8 «Memorandum of understanding between the FASB and the IASB ». La version d'octobre 2006 de ce projet précise les éléments de travail suivants : objectifs et caractéristiques qualitatives, éléments de reconnaissance, éléments de mesure, reporting des entités, présentation et diffusion des états financiers, objectifs du cadre conceptuel et hiérarchie des principes comptables généralement admis, l'application aux entités à but non lucratif et la totalité du cadre conceptuel.
} 
- la normalisation de droit et la normalisation de fait (Colette et Richard 2000)

- la normalisation étatique (ou auto-régulation), la normalisation professionnelle et la normalisation par des organismes indépendants (Colasse 2004a)

Le modèle proposé par Nobes (1992) a longtemps servi de référence pour la classification des différents systèmes comptables à travers le monde. La typologie proposée, basée sur six critères (système juridique, organisation des affaires, structure de l'actionnariat, marchés boursiers, profession comptable et événements historiques), classe les modèles selon qu'ils ont une orientation micro ou macro. Ce modèle a été repris par plusieurs autres chercheurs.

En tenant compte du rôle majeur joué actuellement par l'IASB dans la normalisation comptable internationale, nous avons essayé de proposer quatre stratégies de normalisation possibles. Notre proposition se fonde sur deux critères précis :

a- L'intervention de l'Etat et des organismes professionnels nationaux dans la production des normes applicables dans un pays quelconque. La plupart des Etats exerçaient, jusqu'à 1980, leur activité de normalisation dans un cadre strictement national; l'influence de l'étranger était faible. Cette situation a rapidement évolué dans le dernier quart du $20^{\text {ème }}$ siècle avec notamment l'apparition d'organismes de normalisation internationaux (essentiellement l'IASB). Cette intervention est considérée comme forte lorsque l'Etat et/ou les organismes professionnels du pays sont chargés, séparément ou conjointement, de la production des normes applicables par les entreprises du pays. Lorsque leur rôle se limite à l'approbation de normes établies par des organismes étrangers, elle est considérée comme étant faible (parfois nulle).

b- Le recours aux IAS/IFRS dans la formulation des normes applicables. Le recours aux IAS/IFRS est considéré comme faible si les normalisateurs produisent leurs propres référentiels en adoptant un modèle différent de celui de l'IASB ou en s'inspirant d'autres sources de normalisation internationales.

Cette typologie permet d'éviter certaines discordances ou confusions dans la terminologie utilisée pour qualifier la position de certains pays vis-à-vis de l'IASB ${ }^{9}$, et pourrait servir de base pour analyser le parcours de certains pays et de les classer par rapport aux différentes stratégies proposées. En se basant sur ces deux critères, nous pouvons distinguer quatre stratégies possibles.

\footnotetext{
${ }^{9}$ Plusieurs termes sont utilisés pour qualifier la position d'un pays vis -à-vis des normes de l'IASB (adoption, adaptation, alignement, convergence...) qui prêtent à confusion (à titre d'exemple, l'IASB a classé dans le rapport publié en 2003 la Tunisie parmi les pays ayant adopté les normes IAS depuis 1996 alors que l'application de ces normes n'est pas permise dans ce pays. Le système comptable des entreprises tunisiennes est certes fortement inspiré des normes IAS mais il est obligatoire pour toutes les entreprises qu'elles soient cotées ou non cotées).
} 
Figure 1 : Différentes stratégies de normalisation à adopter face à l'IASB

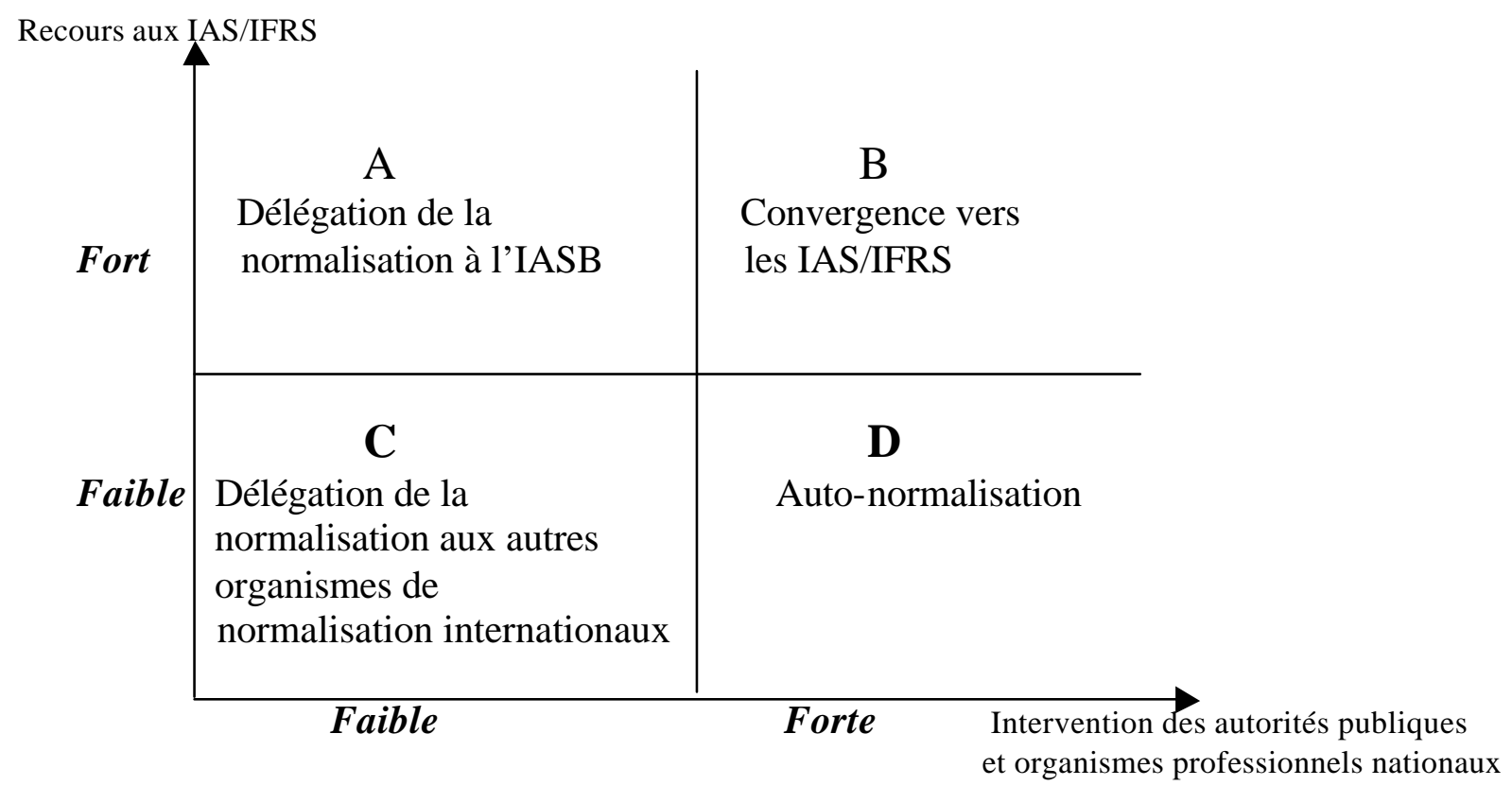

A-Stratégie de délégation de la normalisation à l'IASB : où ni les pouvoirs publics ni les organismes professionnels nationaux ne participent à la normalisation puisque les entreprises dans ce cas doivent se référer directement aux IAS/IFRS. Cela correspond actuellement aux pays faisant partie de l'Union Européenne en ce qui concerne les comptes consolidés des sociétés cotées. Si cette stratégie sera développée à travers le monde, cela pourrait amener à la standardisation des IAS/IFRS.

B-Stratégie de convergence vers les IAS/IFRS : où les pouvoirs publics et/ou les organismes professionnels nationaux produisent des normes qui convergent et se réfèrent aux IAS/IFRS. Tel est le cas de certains pays africains comme la Tunisie. Cette stratégie pourrait conduire à une certaine harmonie au niveau international mais qui reste partielle vue les spécificités de normalisation de chaque pays.

C-Stratégie de délégation de la normalisation à d'autres organismes de normalisation internationaux : où les pouvoirs publics et/ou les organismes professionnels du pays ne jouent aucun rôle (ou jouent un faible rôle d'approbation), la mission de normalisation est confiée à d'autres organismes de normalisation internationaux. Cette stratégie répond généralement à des objectifs économiques et politiques (cas de certains pays d'Afrique avec l'OHADA $^{10}$ et des pays européens avec les directives) et conduit à une certaine harmonisation mais régionale.

\footnotetext{
${ }^{10}$ OHADA: organisation d'harmonisation en Afrique du droit des affaires
} 
D-Stratégie d'auto-normalisation : où les pouvoirs publics et/ou organismes professionnels de chaque pays produisent des normes sans se référer aux IAS/IFRS, qui leur sont propres (c'était la stratégie longtemps préconisée par certains pays comme l'Allemagne et la France) ou qui sont fortement inspirés d'autres référentiels. Elle a permis de produire des modèles comptables diversifiés notamment le modèle continental par opposition au modèle anglosaxon.

Nous avons récapitulé dans le tableau présenté en annexe 1 les caractéristiques, les avantages et les inconvénients liés à chacune de ces stratégies. Dans ce qui suit, notre analyse sera approfondie et appuyée par Pétude du parcours suivi par la France et principalement de la stratégie de convergence vers les IAS/IFRS adoptée ces dernières années.

\subsection{L'expérience française : D-C-B-A}

A la différence des pays anglo-saxons, la normalisation comptable a été confiée en France depuis la deuxième guerre mondiale à l'Etat. Une commission de normalisation des comptabilités a été instituée en 1946 pour élaborer le PCG de 1947 (stratégie D). Elle a été remplacée ensuite par le Conseil Supérieur de la Comptabilité puis par le CNC en 1957. Même si la normalisation, à ce stade, se limitait à la proposition d'un plan détaillé des comptes, elle transmettait une certaine philosophie comptable qui correspondait au modèle continental par opposition au modèle anglo-saxon. Parmi les caractéristiques de ce modèle, la dominance du principe du coût historique, de patrimonialité et de prudence et l'influence remarquable des règles fiscales. La normalisation comptable en France a connu ensuite une phase d'harmonisation à l'échelle européenne avec les directives (stratégie C). Ces dernières n'avaient pas la force des lois qui s'appliquent immédiatement dans chaque Etat membre, elles ne sont applicables que lorsque l'Etat, dans un délai fixé par la directive elle-même, a introduit son contenu dans sa propre réglementation. En France, le contenu de la quatrième directive fût intégré dans la législation par une loi de 1983, celui de la septième par une loi de 1985. Le PCG fût modifié en conséquence. Ces directives sont le fruit d'un compromis entre deux grandes conceptions de la comptabilité : la conception britannique et la conception franco-allemande. Les apports les plus marquants de ces directives était l'introduction de la notion d'image fidèle et la remise en cause du principe de patrimonialité. En 1998, un nouvel organisme, le CRC, adjoint au CNC a été crée. Tous les avis du CNC, qui élabore les normes, ne deviennent pas des règlements. C'est le CRC qui transforme certains avis en règlements, lesquels une fois approuvés par arrêté du ministre de l'économie et des finances se trouvent 
inscrits dans le droit comptable. Mais le véritable «big bang » comptable pour la France et tous les autres pays faisant partie de l'Union était l'adoption des normes de l'IASB depuis 2005. En effet, les entreprises françaises cotées en bourse sont obligées d'établir leurs comptes consolidés conformément aux normes internationales de l'IASB (stratégie A). Ce passage a entraîné selon Chiapello (2005) un bouleversement de tous les concepts comptables, de nouveaux principes comptables et une nouvelle hiérarchie entre principe, une nouvelle conception de l'entreprise et des utilisateurs de l'information comptable. Une série des différentes discordances entre les deux référentiels, français et international, a été dressé par Delessalle (2002). Afin de faciliter ce passage, la normalisation comptable en France passe ces dernières années par une phase transitoire qui a pour objectif de rapprocher le DCF des IAS/IFRS (stratégie B). En effet, dans la plupart des pays de l'Union Européenne, dont la France, les comptes sociaux des sociétés cotées et les comptes des sociétés non cotées restent soumis au référentiel national. Cependant l'application obligatoire des IAS/IFRS aux comptes consolidés des sociétés cotées entraîne une véritable tension sur les droits comptables nationaux qui sont alors incités à converger vers les solutions des IAS/IFRS. C'est ainsi qu'en France, le législateur a entrepris un certain nombre de modifications à la réglementation comptable afin de se rapprocher des IAS/IFRS. Le CNC a évoqué depuis 2001, au cours du processus d'élaboration du règlement européen $n^{\circ} 1606 / 2002$, «sa forte volonté de convergence des comptes consolidés et individuels avec les normes IAS, il paraît difficilement concevable de maintenir deux référentiels à terme. D'où l'objectif de faire évoluer le règlement $n^{\circ}$ 99-03 relatif au PCG avec les normes IAS, tout en aménageant un système d'information simplifié pour les PME et TPE». Et depuis, les avis du CNC et les règlements du CRC ont contribué à modifier le DCF. Parmi les principaux textes allant dans ce sens :

- le règlement du CRC 2000-06 du 7 décembre 2000 relatif aux passifs inspiré de la norme IAS 37,

- le règlement du CRC 2002-10 du 12 décembre 2002 relatif à l'amortissement et à la dépréciation des actifs, inspiré des normes IAS 16 et IAS 36,

- les avis du CNC n²004-15 du 23 juin 2004 et n²004-05 du 25 mars 2004 relatifs à la définition, la comptabilisation et l'évaluation des actifs inspirés des normes IAS16, IAS 38, IAS 2 et IAS 23. 
Parallèlement aux évolutions de la réglementation comptable, plusieurs groupes de travail ont été constitués auprès du $\mathrm{CNC}$ ayant pour objectif de réfléchir aux évolutions à moyen terme de la comptabilité française ${ }^{11}$.

Dans ce sui suit, nous allons essayer d'analyser l'efficacité de ces mesures à travers l'analyse de la comparabilité des données comptables publiées par 30 entreprises françaises ayant opté pour une double présentation de leurs états financiers selon les deux référentiels français et international en 2004 et 2005. Une revue de la littérature sur les principaux travaux réalisés en matière d'harmonisation comptable internationale précède la présentation des résultats de cette étude.

\section{REVUE DE LA LITTERATURE}

Les travaux sur l'harmonisation comptable internationale menée par l'IASB sont de plus en plus nombreux et sont dans la majorité des cas appuyés par des études empiriques (AbdElsalam et Weetman 2003). Le développement de recherches et d'outils de mesure permettant la comparaison entre différents référentiels comptables est nécessaire selon Fontes et al. (2005) pour deux raisons principales. La première raison a trait au nombre croissant de pays ayant décidé de converger vers les normes de l'IASB. La deuxième raison concerne certains pays qui font la combinaison entre plusieurs référentiels. En effet, dans la majorité des pays européens, les sociétés cotées en bourse présentent leurs états financiers consolidés selon un référentiel différent de celui qui sert à la présentation de leurs comptes sociaux et aux comptes des sociétés non cotées. Pour ce genre de pays comme pour les autres, il serait avantageux de permettre aux lecteurs des états financiers d'évaluer la qualité des informations comptables publiées selon différents référentiels et d'expliquer les différences qui pourraient exister.

Les recherches antérieures ont essayé de comparer et de mesurer la convergence de différents systèmes comptables avec celui de l'IASB :

- les normes américaines US GAAP (Larson et Street 2004, Street et al. 2002, Haverty 2006, Emenyonu et Gray 1996)

- les normes comptables dans certains pays européens (Delvaille et al. 2005, Fontes et al. 2005, Emenyonu et Gray 1992, 1996, Weetman et al. 1998, Haller et Eierle 2004)

\footnotetext{
${ }^{11}$ Parmi ces groupes crées en 2003:

- le groupe «IAS et fiscalité » afin d'étudier les incidences fiscales du passage aux IAS/IFRS

- le groupe «IAS et PME» afin d'étudier les possibilités d'application des normes IAS/IFRS aux PME

- le groupe «IAS et droit » afin d'étudier les incidences juridiques du passage aux IAS/IFRS
} 
- $\quad$ autres référentiels ((Abd-Elsalam et Weetman 2003, Emenyonu et Gray 1996)

Au niveau européen, la majorité des recherches ont mis l'accent sur la contribution de l'IASB à la réalisation de la convergence comptable internationale. Nous avons pu distingué trois types de recherches.

Le premier type concerne l'adoption volontaire des IAS/IFRS par certaines entreprises européennes et les facteurs qui pourraient l'expliquer. Ortiz (2005) a conclu, à travers une étude menée sur une période allant de 1997 à 2000, que les sociétés cotées sur le NYSE préfèrent publier leurs états financiers selon les normes américaines lorsque la réglementation de leurs pays leur permet d'adopter un référentiel comptable différent du référentiel national. Mais cette situation a changé après l'adoption des IAS/IFRS. En effet, depuis 2002, la publication des états financiers selon le référentiel international est devenue de plus en plus fréquente. Mais les recherches empiriques dans ce cadre offrent des résultats mitigés quant à l'amélioration de la qualité des informations produites (Street et al. 1999, Taylor et Jones 1999). Parmi les facteurs qui ont une influence sur la publication volontaire des états financiers selon le référentiel international, sans qu'il ait d'obligations légales, nous pouvons citer la taille, la diversification internationale et la structure du capital (Dumontier et Raffounier 1998), le ratio d'endettement (El Gazzar et al. 1999), le chiffre d'affaires à l'étranger et la cotation à l'étranger (Tarca 2004, El Gazzar et al. 1999), la préférence des managers pour les normes internationales (Glaum 2000), les auditeurs (Street et Gray 2002) et la dispersion géographique des affaires (El Gazzar et al. 1999).

Le deuxième type de recherche traite des conséquences et des problèmes liés à la convergence comptable internationale en Europe (Haller 2002, Haller et Eierle 2004, Nobes et Parkers 2004, Stolowy et Jeny-Cazavan 2001, Street et Larson 2004). Parmi ces problèmes, l'intégration de la notion de «true and fair view» en Europe (Aisbitt et Nobes 2001, Alexander et Archer 2003, Evans 2003). Selon Street et Larson (2005), les obstacles qui peuvent limiter cette convergence sont l'influence des règles fiscales (Guenther et Hussein 1995, Meek et Thomas 2004, Eberhartinger 1999, Holeckova 1996, Jaruga et al. 1996, Lamb et al. 1998) et la complexité de certaines règles prévues dans les normes surtout celles en relation avec la juste valeur. Ces auteurs ont conclu dans leur étude que la majorité des pays européens n'envisagent pas de faire converger leurs propres référentiels vers les IAS/IFRS, d'où l'émergence d'une nouvelle forme de «cohabitation» entre plusieurs référentiels dans un même pays. Haller (2002) considère que cette situation ne permet en aucun cas l'harmonisation des règles comptables. Une étude menée par Jermakowicz et Gornick (2006) auprès de 410 entreprises européennes cotées a montré que la majorité de ces entreprises a 
appliqué les normes IAS/IFRS uniquement pour la préparation de leurs comptes consolidés. Le processus lié au passage du référentiel national au référentiel international est considéré comme coûteux et complexe. Parmi les difficultés soulevées lors de cette enquête, la complexité des normes IAS/IFRS et l'absence d'un guide qui pourrait orienter les entreprises. Les auteurs ont concluent que la majorité de ces entreprises n'avaient pas l'intention de procéder à l'application des normes internationales si ces dernières n'étaient pas obligatoires.

Le troisième type de recherche s'intéresse à la convergence formelle (Tay et Parker 1990, Garrido et al. 2002) et à la convergence informelle (Emenyonu et Gray 1992, 1996, Herrmann et Thomas 1995, 1996, Murphy 2000, van der Tas 1988, 1992, Archer et al. 1996, Pierce et Weetman 2002, Aisbitt 2001). Rahman et al. (2002) défendent l'idée que la convergence formelle permet d'améliorer la convergence informelle alors que Schultz et Lopez (2001) ont conclu à travers leur étude que la convergence des normes ne conduit pas nécessairement à une convergence des pratiques surtout si les normes sont complexes et ambiguës. D'une manière générale, ce genre d'études ont posé des problèmes de mesures de la convergence qu'elle soit formelle ou informelle. Les études menées sur la convergence des pratiques ont utilisé les informations publiées dans les rapports annuels pour calculer des indicateurs (Haverty 2006, Canibano et Mora 2000) dont les plus utilisés étaient ceux de van der Tass (1988). Des coefficients d'association ont été utilisé par d'Arcy (2001) et des coefficients de Jaccard par Rahman et al. (2002). Quant à la convergence formelle, elle a été analysée par des analyses discriminantes (Rahman et al. 1996), par le calcul des distances euclidiennes (Garrido et al. 2002) et par d'autres mesures ${ }^{12}$.

Les études menées sur l'harmonisation formelle et informelle au niveau européen ne sont pas nombreuses (Emenyonu et Gray 1992, 1996, Fontes et al. 2005, Haller et Eierle 2004, Weetman et al. 1998, Delvaille et al. 2005) et peu d'entre elles s'est intéressé au cas français (Ding et al. 2005). Notre étude tend à comparer les données comptables produites par les entreprises françaises en 2004 et 2005 selon les deux référentiels français et international et d'établir une éventuelle relation entre la comparabilité des résultats et certaines caractéristiques des ent reprises.

\footnotetext{
12 coefficients de Jaccard et Spearman par Fontes et al. 2005
} 


\section{DE L'EFFICACITE DES MESURES DE CONVERGENCE VERS LES IAS/IFRS EN FRANCE}

\subsection{Hypothèses et méthodologie de recherche}

Nous considérons que les modifications apportées au DCF ont été efficaces si les données comptables (par exemple le résultat net) obtenues à partir de l'application du DCF et des IAS/IFRS seraient comparables. Des informations comptables sont comparables si elles sont similaires de telle manière que le lecteur des états financiers puisse, à travers un certain nombre d'ajustements passer d'un référentiel à un autre (Haverty 2006). En effet, le passage aux IAS/IFRS conduit les groupes français à procéder à un certains nombre :

- de reclassements traduisant des différences de présentation des comptes du bilan, du compte de résultat et du tableau des variations des flux de trésorerie,

- de retraitements induits par les différences de méthodes de valorisation ayant pour conséquences des incidences sur les réserves et les résultats consolidés.

Compte tenu des mesures de convergence entreprises depuis quelques années afin d'éviter ces différences, nous avons supposé que les résultats obtenus selon les deux référentiels sont comparables.

\subsubsection{Choix de l'échantillon}

L'échantillon comporte 30 entreprises françaises cotées en bourse et concernées par le règlement européen ${ }^{13}$. Nous n'avons retenu que celles ayant procédé à une double présentation de leurs états financiers selon le DCF et les IAS/IFRS. Les données ont été collectées à travers les états financiers annuels ou les rapports spéciaux publiés de façon indépendante et consacrés à l'analyse du passage aux normes IAS/IFRS. L'étude a concerné les années 2004 et 2005 et n'a pas pu être étendue à d'autres exercices puisque la majorité des entreprises ne procèdent qu'une seule fois à cette double présentation des états financiers. Qu'ils s'agissent d'informations obligatoires ou volontaires ${ }^{14}$, l'objectif de notre travail est de procéder à une analyse comparative des données comptables obtenues à l'aide de deux référentiels différents à savoir le DCF et les IAS/IFRS.

\footnotetext{
13 Annexe 2

${ }^{14}$ Pour l'exercice 2004, les états financiers présentés selon les IAS/IFRS sont publiés de façon volontaire par certaines entreprises afin d'anticiper le passage en 2005.
} 


\subsubsection{Mesure des variables}

Nous avons utilisé dans notre analyse les indices de la comparabilité et de la convergence de Gray (1980) et de Haverty (2006).

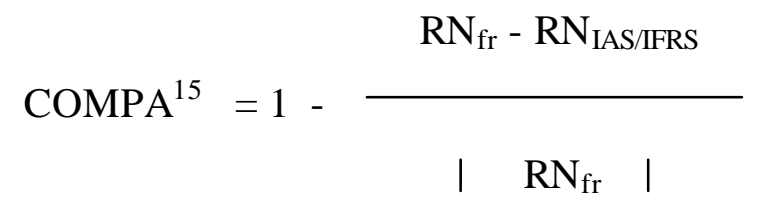

Cet indice présente l'avantage de pouvoir fournir une idée sur les différences qui existent entre les résultats calculés selon les deux référentiels, mais peut présenter des valeurs extrêmes si le dénominateur est important. Si les résultats sont comparables, l'indice tend vers 1 au seuil de 5\%. Nous avons ensuite utilisé les mêmes indices développés par Haverty (2006).

Variation du résultat net : $\mathrm{VAR}_{\mathrm{RN}}=\frac{\left|\mathrm{RN}_{\mathrm{fr}}-\mathrm{RN}_{\mathrm{IAS} / \mathrm{IFR} \mathrm{S}}\right|}{\mathrm{AN}_{\mathrm{fr}}}$
Variation de l'actif net : $\mathrm{VAR}_{\mathrm{AN}}=\frac{\left|\mathrm{AN}_{\mathrm{fr}}-\mathrm{AN}_{\mathrm{IAS} / \mathrm{IFRS}}\right|}{\mathrm{AN}_{\mathrm{fr}}}$

Variation de la rentabilité des capitaux propres : $\mathrm{VAR}_{\mathrm{RCP}}=\left|\mathrm{RCP}_{\mathrm{fr}}-\mathrm{RCP}_{\mathrm{IAS} / \mathrm{FRS}}\right|$

Variation du bénéfice par action : $\mathrm{VAR}_{\mathrm{BA}}=\frac{\mid \mathrm{BA}_{\mathrm{fr}}-\mathrm{BA} \text { IAS/IFRS }}{\mid}$

$\mathrm{BA}_{\mathrm{fr}}$

Les résultats sont considérés être comparables si ces indices tendent vers 0 au seuil de $5 \%$.

Avec :

$\mathrm{RN}_{\mathrm{fr}}$ : résultat net selon le DCF

$\mathrm{RN}_{\text {IAS/IFRS }}$ : résultat net selon les IAS/IFRS

$\mathrm{AN}_{\mathrm{fr}}$ : actif net selon le $\mathrm{DCF}$

$\mathrm{AN}_{\text {IAS/IFRS }}$ : actif net selon les IAS/IFRS

$\mathrm{RCP}_{\mathrm{fr}}$ : rentabilité des capitaux propres selon le DCF

$\mathrm{RCP}_{\text {IAS/IFRS }}$ : rentabilité des capitaux propres selon les IAS/IFRS

$\mathrm{BA}_{\mathrm{fr}}$ : bénéfice par action selon le DCF

$\mathrm{BA}_{\text {IAS/IFRS }}$ : bénéfice par action selon les IAS/IFRS

${ }^{15}$ cet indice a été également utilisé dans les études de Weetman et al. (1998), Street et al. (2000) et Haverty (2006) 


\subsection{Interprétation des résultats}

\subsubsection{Analyse de la comparabilité des informations comptables publiées selon les deux référentiels français et international}

Les résultats obtenus pour l'indice COMPA ${ }^{16}$ montrent que quatre entreprises présentent des résultats comparables au seuil de 5\%. Cela signifie que $87 \%$ de l'échantillon présentent des résultats non comparables malgré les mesures de convergence entreprises.

L'indice de comparabilité le plus élevé est celui de PROSODIE $(4,42)$, pour cette entreprise donc, le résultat calculé selon le référentiel international est quatre fois plus supérieur que celui calculé selon le DCF. En revanche, le résultat IAS/IFRS d'OPERA CONSTRUCTION est légèrement supérieur au résultat français (différence inférieure à 1\%).

L'annexe 2 résume également les résultats obtenus pour les indices de Haverty (2006) :

- La variation du résultat $\left(\mathrm{VAR}_{\mathrm{RN}}\right)$ permet de conclure que $80 \%$ des entreprises de l'échantillon ont des résultats comparables.

- La variation de l'actif net $\left(\mathrm{VAR}_{\mathrm{AN}}\right)$ permet de conclure que $53 \%$ des entreprises de l'échantillon ont des indices comparables.

- La variation de la rentabilité des capitaux propres $\left(\mathrm{VAR}_{\mathrm{RCP}}\right)$ permet de conclure que $80 \%$ des entreprises de l'échantillon ont des indices de rentabilité comparables.

- La variation du bénéfice par action $\left(\mathrm{VAR}_{\mathrm{BA}}\right)$ permet de conclure que $10 \%$ des entreprises l'échantillon ont des bénéfices par actions comparables.

Nous pouvons ainsi conclure que les indices de comparabilité relatifs aux résultats nets, à l'actif net et à la rentabilité des capitaux propres montrent que la majorité des entreprises vérifient l'hypothèse de comparabilité. En revanche, plus de $90 \%$ des entreprises présentent des bénéfices par actions selon les deux référentiels qui ne peuvent pas être considérés comme comparables.

Aucune entreprise ne permet de confirmer l'hypothèse de comparabilité à l'aide des quatre indices calculés, en revanche trois entreprises permettent de réfuter cette hypothèse à l'aide de tous les indices qui sont non significatifs.

\subsubsection{Comparabilité/absence de comparabilité et caractéristiques des entreprises}

En analysant les comptes de résultats des différentes entreprises, nous avons pu constater que les principaux retraitements sont dus aux éléments suivants :

\footnotetext{
${ }^{16}$ Annexe 3
} 
- Les contrats de location: selon l'IAS 17, il faut inscrire à l'actif du bilan du locataire la valeur des biens utilisés au titre d'un contrat de location financement, alors que selon le DCF, cette pratique est interdite dans les comptes individuels et préférentielle dans les comptes consolidés.

- Les avantages du personnel : selon l'IAS 19, il faut comptabiliser une provision pour engagement de retraite du personnel, alors que selon le DCF, cette inscription est préférentielle.

- L'évaluation des instruments financiers : l'IAS 39 stipule que les titres de placement doivent être valorisés à la juste valeur, alors que selon le DCF, les moins values font l'objet d'une dépréciation les plus values ne sont pas comptabilisées.

- L'amortissement de l'écart d'acquisition : permis par le DCF mais interdit par l'IFRS 3 qui a instauré l'obligation d'effectuer des tests de valeur systématique selon la méthode des cash flow actualisés préconisés par la norme IAS 36.

- Les charges à répartir : comptabilisées comme actif fictif dans le DCF, doivent être annulées selon l'IAS 38 .

- Les actions propres : comptabilisées parmi les valeurs mobilières de placement sont reclassées en capitaux propres selon les normes IAS 32 et 39.

Compte enu des résultats obtenus, nous avons cherché à faire la correspondance entre la comparabilité ou l'absence de comparabilité des états financiers avec la taille et le secteur d'activité.

Des études de corrélation par des équations de régressions simples ont été réalisées avec les variables suivantes :

- Pour la taille des entreprises : nous avons choisi comme variable de mesure le chiffre d'affaires $(\mathrm{CA})$ réalisé au cours de l'année.

- Pour le secteur d'activité : nous avons fait la distinction entre les banques et autres institutions financières, d'une part, et les sociétés industrielles commerciales et de services d'autre part. La variable correspondante (SECT) prend la valeur 1 pour le premier type d'entreprises et 0 sinon.

L'analyse des résultats ${ }^{17}$ de la relation entre les indices de comparabilité et les caractéristiques des entreprises permettent de conclure que la variable relative au chiffre d'affaires CA est positivement corrélée avec les indices COMPA, VAR $R_{R N}$ et $V_{A} R_{R C P}$. Cela signifie que plus l'entreprise est grande plus les résultats obtenus selon les deux référentiels français et

\footnotetext{
${ }^{17}$ Annexe 5
} 
international sont comparables. Les petites entreprises sont plus amenées à faire des retraitements et des modifications pour la transition des comptes. Ainsi leurs résultats semblent peu comparables. La variable relative au secteur d'activité SECT s'est avérée non significative pour toutes es mesures de la comparabilité, ce qui permet de conclure que les entreprises sont confrontées aux mêmes problèmes de retraitements quel que soit leur secteur d'activité.

\section{CONCLUSION}

Ce papier a permis d'analyser le parcours de la France qui se trouve actuellement fortement influencée par les normes de l'IASB en matière de normalisation comptable. Malgré les mesures entreprises en France depuis quelques années pour faciliter le passage des grandes firmes cotées et pour aligner le DCF aux IAS/IFRS, cette étude a permis de conclure que les données comptables présentées par une trentaine d'entreprises selon les deux référentiels national et international sont peu comparables. Ce résultat est plus remarquable dans les petites entreprises.

La France doit ainsi continuer à faire des efforts pour rapprocher son droit comptable des solutions adoptées par les IAS/IFRS. Mais les véritables questions qui se posent : jusqu'à quel point et quelles risques présentent cette stratégie de convergence?

Le débat reste ouvert sur le rythme de ce processus de convergence alors que le référentiel de l'IASB est lui-même en évolution, sur le coût de ce processus pour l'Etat et pour les entreprises, sur les véritables attentes des différents utilisateurs des informations comptables, sur les problèmes pratiques et les conséquences de l'application de la juste valeur, sur la position des autres pays européens...

Cette étude présente des limites notamment au niveau du choix de l'échantillon, du choix des mesures et de la période étudiée. L'étude limitée au cas de la France est certes intéressante et d'actualité, mais reste insuffisante dans la mesure où elle ne permet pas, elle seule, de valider la typologie de stratégies de normalisation proposée. En effet, l'analyse devra être appuyée par d'autres cas. En 2005, l'IASB a remporté une longue et difficile bataille mais n'a pas gagné la guerre. Il est évident qu'il va marquer pendant de longues années les systèmes comptables dans différents continents, mais le véritable enjeu qui va déterminer le sort des normes de l'IASB est la stratégie future du FASB. Le «break» annoncé par l'IASB a pour objectif d'assurer une certaine stabilité jusqu'à 2009 et d'assister ceux qui souhaitent 
implanter les IAS/IFRS à travers le monde ${ }^{18}$. L'analyse du parcours de la normalisation comptable aux Etats Unis paraît ainsi une nouvelle piste de recherche intéressante.

\footnotetext{
${ }^{18}$ voir le site de l'IASB, www.iasb.org « No major standards to be effective before 2009»
} 


\section{BIBLIOGRAPHIE}

Abd Esalam O.H. et Weetman P. (2003), «Introducing International Accounting Standards to an emerging capital market: relative familiarity and language effect in Egypt», Journal of International Accounting, Auditing and Accountability, ${ }^{\circ}$ 12, pp. 63-84.

Achleitner A. (1995), «Latesd developments in Swiss regulation of financial reporting », The European Accounting Review, Vol. 4, n 1, pp. 141-154.

Aisbitt S. (2001), «Measurement of harmony of financial reporting within and between countries: the case of Nordic countries », The European Accounting Review, Vol. 10, n 1, pp. 51-72.

Aisbitt S. et Nobes C. (2001), « The true and fair view requirement in recent national implementations », Accounting and Business Research, Vol. 31, n 2, pp. 83-90.

Amblard M. (2004), «Conventions et comptabilité : vers une approche sociologique du modèle », Comptabilité, Contrôle et Audit, numéro spécial, pp. 47-68.

Alexander D. et Archer S. (2003), «On economic reality, representational faithfulness and the true and fair override », Accounting and Business Research, Vol. 33, n 1, pp. 3-17.

Alexander D. et Schwencke H.R. (2003), «Accounting changes in Norway», The European Accounting Review, Vol. 12, n 3, pp. 549-566.

Archer S., Delvaille P. et McLeay S. (1996), «A statistical model of international accounting harmonisation », Abacus, Vol. 32, n 1, pp. 1-29.

Barbu E. (2004), « 40 ans de recherche en harmonisation comptable internationale », $25^{\text {ème }}$ congrès de l'AFC, Orléans, France.

Belkaoui A. (1984), Théorie comptable, Presses de l’Université du Québec.

Blic D. (2004), «La comptabilité à l'épreuve du scandale financier », Comptabilité, Contrôle et Audit, numéro spécial, pp. 7-28.

Canibano L. et Mora A. (2000), «Evaluating the statistical significance of de facto accounting harmonisation: A study of European global players », The European Accounting Review, Vol. 9, $n^{\circ} 3$, pp. 349-369.

Colasse B. (2000), «Harmonisation comptable internationale », dans Colasse B. Encyclopédie de Comptabilité, Contrôle de gestion et Audit, Economica, Paris, pp. 757-769.

Colasse B. (2002), « La guerre des normes n’aura pas lieu », Sociétal, n³7, $3^{\text {ème }}$ trimestre, pp. 89-93.

Colasse B. (2004a), «La régulation comptable : entre privé et public », Conférence prononcée le 13 septembre 2004 dans le cadre du séminaire «Nouvelles normes comptables : quels enjeux pour l'enseignement de la comptabilité » organisé par l'Université de Paris Dauphine.

Colasse B. (2004b), « La résistible ascension de l'IASC/IASB », Gérer et Comprendre, pp. 30-40.

Collette C. et Richard J. (2000), Comptabilité générale : les systèmes français et anglo-saxons, DUNOD, Paris.

Chiapello E. (2005), «Les normes comptables comme institution du capitalisme : une analyse du passage aux normes IFRS en Europe à partir de 2005 », Sociologie du Travail, n 47, pp. 362-382.

Choi F.D., Frost C.A. et Meek G.K. (2001), International accounting, Pearson Education.

d'Arcy A. (2001), «Accounting classification and the international harmonisation debate, an empirical investigation », Accounting, Organizations and Society, $n^{\circ}$ 26, pp. 327-349. 
Delesalle E. (2002), «La comptabilité du XXIème siècle se prépare... », $23^{\text {ème }}$ Congrès de l'AFC, Toulouse, France.

Delvaille P., Ebbers G. et Saccon C. (2005), « International financial reporting convergence: evidence from three continental European countries », Accounting in Europe, Vol. 2, pp. 137-164.

Ding Y., Jeanjean T. et Stolowy H. (2005), «Why do national GAAP differ from IAS? The role of culture », The International Journal of Accounting, $n^{\circ} 40$, pp. 325-350.

Dumontier P. et Raffounier B. (1998), «Why firms comply with voluntary with IAS: an empirical analysis with Swiss data », Journal of International Financial Management and Accounting, Vol. 9, ${ }^{\circ}$ 3, pp. 216-245.

Eberhartinger E.L.E. (1999), «The impact of tax rules on financial reporting in Germany, France and UK », The International Journal of Accounting, Vol. 34, n 1, pp. 93-119.

Eilifsen A. (1996), «The relationship between accounting and taxation in Norway », The European Accounting Review, , $\mathrm{n}^{\circ}$ 5, pp. 835-844.

El Gazzar S., Finn P., et Jacob R. (1999), «An empirical investigation of multinational firms' compliance with International Accounting Standards », The International Journal of Accounting, Vol. (34), nº 2, pp. 239-248.

Emenyonu E. N., et Gray S. J. (1992), «EC accounting harmonization: An empirical study of measurement practices in France, Germany and the U.K », Accounting and Business Research, Vol. 23 , pp. 89.

Emenyonu E. N., et Gray S. J. (1996), « International accounting harmonization and the major developed stock markets countries: An empirical study », International Journal of Accounting, Vol. 31, p. 3 .

Evans L. (2003), «The true and fair view and the fair representation override of IAS1 », Accounting and Business Research, Vol. 33, ${ }^{\circ}$ 4, pp. 311-325.

FASB (2006), «Conceptual Framework : joint project of the IASB and FASB », www.fasb.org

FASB (2006), «Conceptual Framework for financial reporting: objectives of financial reporting and qualitative characteristics of decision-useful financial reporting information », www.fasb.org

Fontes A., Rodrigues L.L. et Craig R. (2005), « Measuring convergence of national accounting standards with international financial reporting standards », Accounting Forum, n ${ }^{\circ} 29$, pp. 415-436.

Garrido P., Leon A. et Zorio A. (2002), « Measurement of formal harmonization progress : the IASC experience », The International Journal of Accounting, $n^{\circ} 37$, pp. 1-26.

Glaum M. (2000), « Bridging the GAAP: The changing attitude of German managers towards AngloAmerican accounting and accounting harmonization », Journal of International Financial Management and Accounting, Vol. 11, $\mathrm{n}^{\circ}$ 1, pp. 23-47.

Guenther D.A. et Hussein M.E.A. (1995), « Accounting standards and national tax laws: the IASC and the ban on LIFO », Journal of Accounting and Public Policy, ${ }^{\circ}$ 14, pp. 115-141.

Gray S.J. (1980), «The impact of international accounting differences from a security-analysts perspective: some European evidence », Journal of Accounting Research, Vol. 18, Spring, pp. 64-76.

Haller A. (2002), «Financial accounting developments in the European Union : Past, events and future prospects », The European Accounting Review, Vol. 11, n 1, pp. 153-190

Haller A. et Eierle B. (2004), «The adaptation of German accounting rules to IFRS: a legislative balancing act », Accounting in Europe, Vol. 1.

Haverty J.L. (2006), « Are IFRS and US GAAP converging ? Some evidence from People's Republic of China companies listed on the New York Stock Exchange », Journal of International Accounting, Auditing and Taxation, Vol. 15, pp. 48-71. 
Heem G. et Aonzo P. (2003), «La normalisation comptable internationale: ses acteurs, sa légitimité, ses enjeux », Revue d'Economie Financière, n ${ }^{\circ}$ 71, pp.33-61.

Hermann D. et Thomas W. (1995), «Harmonisation of accounting measurement practices in the European community », Accounting and Business Research, Vol. 25, n 100, pp. 253-265.

Herrmann D., et Thomas W. (1996), « Segment reporting in the European Union: Analyzing the effects of country, size, industry, and exchange listing », Journal of International Accounting, Auditing \&Taxation, Vol. 5, $\mathrm{n}^{\circ} 1$.

Holeckova J. (1996), «Relationship between accounting and taxation in the Czech Republic », The European Accounting Review, n ${ }^{\circ}$ 5, pp. 859-869.

Hussey R. et Ong A. (2005), International financial reporting standards desk reference, John Willey and Sons.

IASB (2006), « No new major standards to be effective before 2009 », www.iasb.org

Jaruga A., Walinska E. et Baniewicz A. (1996), « The relationship between accounting and taxation in Poland », The European Accounting Review, n 5, pp. 883-897.

Jermakowicz E.K. et Gornick T.S. (2006), «Implementing IFRS from the perspective of EU publicly traded companies », Journal of International Accounting, Auditing and Taxation, Vol. 15, pp. 170196.

Lamb M., Nobes C. et Roberts A. (1998) «International variations in the connections between tax and financial reporting », Accounting and Business Research, $n^{\circ}$ 28, pp. 173-188.

Larson R.K. et Kenny S.Y. (1999), «The harmonisation of international accounting standards : progress in 1990s? », Multinational Business Review, Vol. 7, $\mathrm{n}^{\circ}$ 1, pp.1-11.

Larson R.K. et Street D.L. (2004), «Convergence with IFRS in an expanding Europe: progress and obstacles identified by large accounting firms' survey », Journal of International Accounting, Auditing and Taxation, Vol. 13, pp.89-119.

Meek G.K. et Thomas W.B. (2004), «A review of markets-based international accounting research », Journal of International Accounting Research, Vol. 3, n 1, pp. 21-41.

Murphy A.B. (2000), "The impact of adopting International Accounting Standards on the harmonisation of accounting practices », The International Journal of Accounting, Vol.35, $\mathrm{n}^{\circ} 4$, pp. 471-493.

Nobes C. (1992), International Classification of Financial Reporting, Routledge, 2ed.

Nobes, C. W., et Parker, R. (1981), Comparative international accounting, IL, Richard D Irwin.

Nobes, C. W., et Parker, R. (2004), Comparative international accounting (8th ed.). London: Prentice Hall.

Ortiz E. (2005), «GAAP choice by European companies », European Business Review , Vol. 17, n 1 .

Pierce A. et Weetman P. (2002), «Measurement of de facto harmonisation: implications of non disclosure for research planning and interpretation », Accounting and Business Research, Vol. 32, $\mathrm{n}^{\circ}$ 4, pp. 259-273.

Prather-Kinsey J. (2006), «Developing countries converging with developed-country accounting standards: evidence from South Africa and Mexico », The International Journal of Accounting, Vol. 41, pp. 141-162.

Rahman A., Perera H. et Ganesh S. (1996), « Measurement of formal harmonisation in accounting: an exploratory study », Accounting and Business Research, Vol. 26, n 4, pp. 325-339.

Rahman A., Perera H. et Ganesh S. (2002), « Accounting practice harmony, accounting regulation and firm characteristics », Abacus, Vol. 38, n 1, pp. 46-77. 
Schipper K. (2005), « The introduction of International Accounting Standards in Europe: implications for international convergence », The European Accounting Review, Vol. 14, n 1, pp. 101-126.

Schultz J., et Lopez T. (2001), «The impact of national influence on accounting estimates: Implications for international accounting standard-setters», The International Journal of Accounting, n 36 , pp. 271-290.

Stolowy H. et Jeny-Cazavan A. (2001), «International accounting disharmony : the case of intangibles », Accounting, Auditing and Accountability Journal, $\mathrm{n}^{\circ} .14$.

Street D.L. et Gray S.J. (2002), «Factors influencing the extent of corporate compliance with International Accounting Standards: summary of a research monograph », Journal of International Accounting, Auditing and Taxation, Vol. 11, n 1, pp. 51-76.

Street D.L. et Gray S.J. et Brayant S.M. (1999), «Acceptance and observance of International Accounting Standards », The International Journal of Accounting, Vol. 34, n 1, pp. 11-48.

Street D. et Larson R.K. (2004), «Large accounting firms' survey reveals convergence of two standard system in the European Union », Advances in International Accounting, $n^{\circ} 17$, pp. 1-29.

Street D. et Larson R.K. (2005), «Large accounting firms' survey reveals convergence of two standard system in the European Union », Advances in International Accounting, $\mathrm{n}^{\circ}$ 17, pp. 1-29.

Street D.L. et Nichols N.B. et Gray S.J. (2000), «Assessing the acceptability of international accounting standards in US: an empirical study of the materiality of US GAAP reconciliations by nonUS companies complying with IASC standards », The International Journal of Accounting, Vol. 35, $\mathrm{n}^{\circ}$ 1, pp. 27-63.

Tarca A. (2004), «International convergence of accounting practices: choosing between IAS and U.S. GAAP », Journal of International Financial Management and Accounting, Vol. 15, n 1, pp. 60-91.

Tay J.S. et Parker R. (1990), «Measuring international harmonization and standardization », Abacus, Vol. 26, $\mathrm{n}^{\circ}$ 1, pp. 71-88.

Taylor M.E. et Jones R.A. (1999), «The use of International Accounting Standards terminology, a survey of IAS compliance disclosure », The International Journal of Accounting, Vol. 34, $\mathrm{n}^{\circ}$ 4, pp. 557-570.

van der Tas L.(1988), «Measurement harmonisation of financial reporting practices », Accounting and Business Research, Vol. 18, $\mathrm{n}^{\circ}$ 70, pp. 157-169.

van der Tas L.(1992), «Evidence of EC financial reporting practice harmonisation », The European Accounting Review, ${ }^{\circ}$ 1, pp. 69-104.

Van Hulle K. (1996), «L'harmonisation comptable européenne: une nouvelle stratégie au regard de l'harmonisation internationale », Revue Française de Comptabilité, Février, pp. 29-38.

Weetman P.E., Jones A.E., Adams C.A. et Gray S.J. (1998), « Profit measurement and UK accounting standards: a case of increasing disharmony in relation to US GAAP and IAS's », Accounting and Business Research, Vol. 28, n³, pp.189-208.

\section{Liste des abréviations utilisées}

CE : Commission Européenne

CNC : Conseil National de Comptabilité

CRC : Comité de Réglementation Comptable

DCF: Droit Comptable Français

IASB : International Accounting Standard Board

IAS : International Accounting Standards

IFRS: International Financial Reporting Standards

PCG: Plan Comptable Général

US GAAP : United States' Generally Accepted Accounting Principles 


\section{Annexe 1 : Caractéristiques, avantages et inconvénients des stratégies de normalisation}

\begin{tabular}{|c|c|c|c|c|}
\hline Caractéristiques & A & B & $\mathbf{C}$ & $\mathbf{D}$ \\
\hline $\begin{array}{ll}\text { Approche } & \text { de } \\
\text { normalisation } & \end{array}$ & Privée internationale & $\begin{array}{l}\text { Publique nationale } \\
\text { privée nationale ou } \\
\text { mixte }\end{array}$ & $\begin{array}{l}\text { Privée internationale } \\
\text { ou publique } \\
\text { internationale ou } \\
\text { mixte }\end{array}$ & $\begin{array}{l}\text { Publique nationale } \\
\text { privée nationale ou } \\
\text { mixte }\end{array}$ \\
\hline $\begin{array}{l}\text { Normes par rapport } \\
\text { aux IAS/IFRS }\end{array}$ & Règles identiques & $\begin{array}{l}\text { Etat et/ou organismes } \\
\text { professionnels } \\
\text { nationaux }\end{array}$ & $\begin{array}{l}\text { Autorité publique } \\
\text { internationale et/ou } \\
\text { organismes } \\
\text { professionnels } \\
\text { internationaux autres } \\
\text { que l'IASB } \\
\text { Règles différentes }\end{array}$ & $\begin{array}{l}\text { Etat et/ou } \\
\text { organismes } \\
\text { professionnels } \\
\text { nationaux }\end{array}$ \\
\hline Avantages & 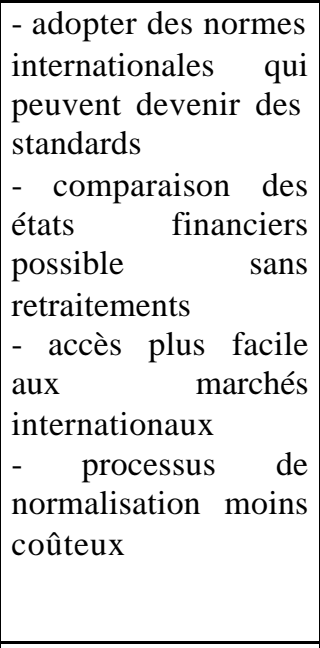 & 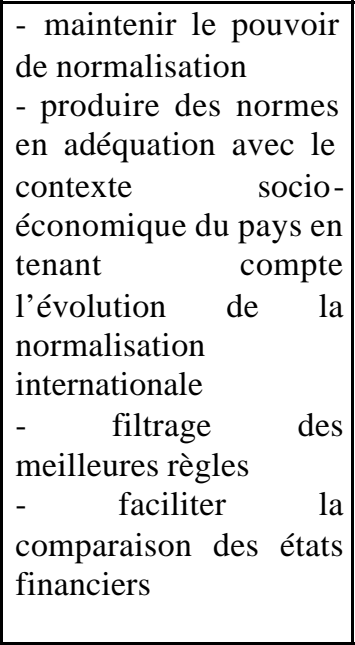 & $\begin{array}{l}\text { - adopter des normes } \\
\text { communes avec } \\
\text { d'autres pays } \\
\text { - répondre à des } \\
\text { enjeux politiques ou } \\
\text { économiques } \\
\text { - comparaison des } \\
\text { états financiers } \\
\text { possible mais limitée } \\
\text { - processus de } \\
\text { normalisation moins } \\
\text { coûteux }\end{array}$ & 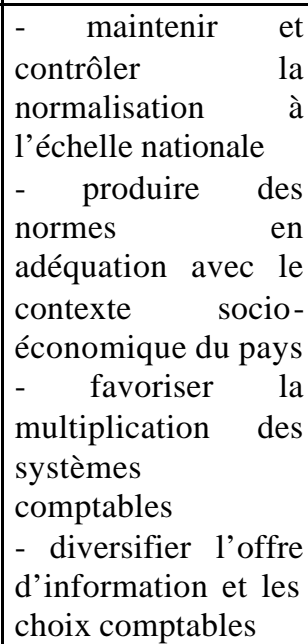 \\
\hline Inconvénients & 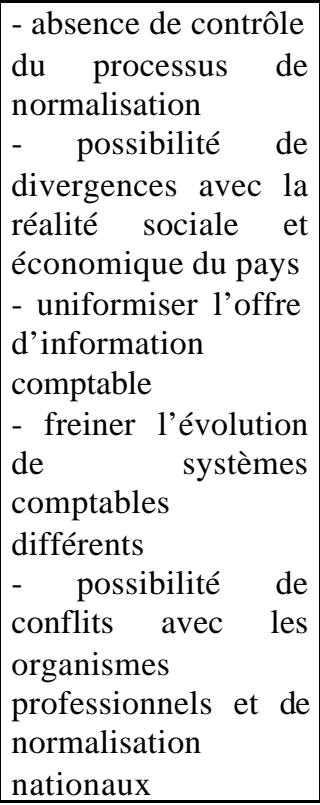 & 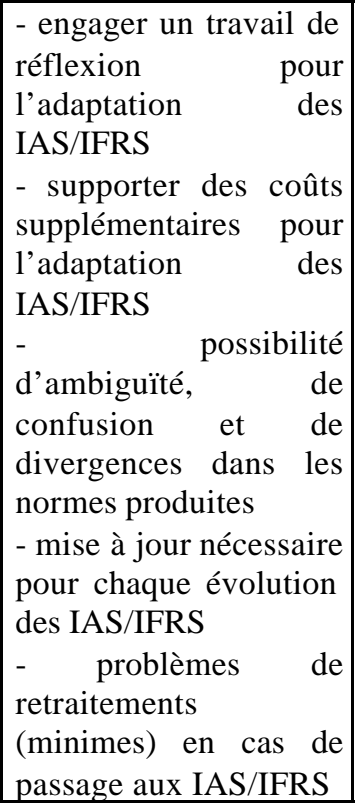 & $\begin{array}{l}\text { - absence de contrôle } \\
\text { du processus de } \\
\text { normalisation } \\
\text { - application souvent } \\
\text { limitée } \\
\text { - possibilité de } \\
\text { divergences avec la } \\
\text { réalité économique } \\
\text { et sociale du pays }\end{array}$ & 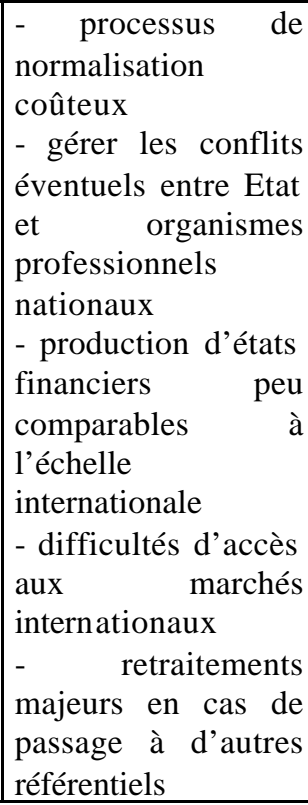 \\
\hline
\end{tabular}


Annexe 2 : Echantillon d'entreprises

\begin{tabular}{|c|l|r|l|}
\hline 1 & ART PRICE & 16 & M6 \\
\hline 2 & AGF & 17 & SOCIETE GENERALE \\
\hline 3 & DELACHAUX & 18 & SILIC \\
\hline 4 & GDF & 19 & VIVENDI \\
\hline 5 & GFI & 20 & VM MATETRIAUX \\
\hline 6 & L'OREAL & 21 & FININFO \\
\hline 7 & MICHELIN & 22 & ALTEN \\
\hline 8 & PAGES JAUNES & 23 & VALEO \\
\hline 9 & PROSODIE & 24 & AUTOROUTES PRR \\
\hline 10 & SIPH & 25 & LVMH \\
\hline 11 & FranCe TELECOM & 26 & TOTAL \\
\hline 12 & OPERA CONSTRUCTION & 27 & AIR France \\
\hline 13 & BOUYGUES & 28 & ILOG \\
\hline 14 & MBRICOLAGE & 29 & REMY COINTREAU \\
\hline 15 & EDF & 30 & OSIATIS \\
\hline
\end{tabular}

Annexe 2 : Indices de comparabilité et de convergence

\begin{tabular}{|c|c|c|c|c|c|c|c|c|c|c|}
\hline & COMPA & & $\mathbf{V A R}_{\mathbf{R N}}$ & & $\mathbf{V A R}_{\mathbf{A N}}$ & & $\mathbf{V A R}_{\mathrm{RCP}}$ & & $\mathbf{V A R}_{\mathbf{B A}}$ & \\
\hline 1 & 1,748285 & $\mathrm{NC}$ & 1,13174 & $\mathrm{NC}$ & 7,19502 & $\mathrm{NC}$ & 1.46599 & $\mathrm{NC}$ & 0.782609 & $\mathrm{NC}$ \\
\hline 2 & 1,207428 & $\mathrm{NC}$ & 0,03108 & $\mathrm{C}^{* *}$ & 0,004207 & $\mathrm{C}^{* * *}$ & 0.03184 & $\mathrm{C}^{* *}$ & 0.20673 & $\mathrm{NC}$ \\
\hline 3 & 0,561968 & $\mathrm{NC}$ & 0,038108 & $\mathrm{C}^{* *}$ & 0,00641 & $\mathrm{C}^{* * *}$ & 0.038419 & $\mathrm{C}^{* *}$ & 0.447917 & $\mathrm{NC}$ \\
\hline 4 & 1,174952 & $\mathrm{NC}$ & 0,01718 & $\mathrm{C}^{* *}$ & 0,01991 & $\mathrm{C}^{* * *}$ & 0.01493 & C** & 0.17241 & $\mathrm{NC}$ \\
\hline 5 & 1,392145 & $\mathrm{NC}$ & 0,0671 & $\mathrm{NC}$ & 0,04179 & $\mathrm{C}^{* * *}$ & 0.07127 & $\mathrm{NC}$ & 0.392145 & $\mathrm{NC}$ \\
\hline 6 & $\begin{array}{ll}1,09487 \\
\end{array}$ & $\mathrm{NC}$ & 0,02947 & $\mathrm{C}^{* *}$ & 0,01079 & $\mathrm{C}^{* * *}$ & 0.02584 & $\mathrm{C}^{* * *}$ & 0.02691 & $\mathrm{C}^{* * *}$ \\
\hline 7 & 1,103343 & $\mathrm{NC}$ & 0,00772 & $\mathrm{C}^{* *}$ & 0,302225 & $\mathrm{NC}$ & 0.04342 & $\mathrm{C}^{* *}$ & 0.10249 & $\mathrm{NC}$ \\
\hline 8 & 0,958063 & $\mathrm{C}^{* *}$ & 0,020701 & $\mathrm{C}^{* * *}$ & 1,03568 & $\mathrm{NC}$ & 0.261311 & $\mathrm{NC}$ & 0.058824 & $\mathrm{NC}$ \\
\hline 9 & 4,427106 & $\mathrm{NC}$ & 0,11611 & $\mathrm{NC}$ & 0,16968 & $\mathrm{NC}$ & 0.10418 & $\mathrm{NC}$ & 3.419355 & $\overline{\mathrm{NC}}$ \\
\hline 10 & 1,208627 & $\mathrm{NC}$ & 0,06536 & $\mathrm{NC}$ & 0,36773 & $\mathrm{NC}$ & 0.036447 & $\mathrm{C}^{* * *}$ & 0.23906 & $\mathrm{NC}$ \\
\hline 11 & 1,083693 & $\mathrm{NC}$ & 0,01486 & $\mathrm{C}^{* *}$ & 0,12767 & $\mathrm{NC}$ & 0.006924 & $\mathrm{C}^{* *}$ & 0.07895 & $\mathrm{NC}$ \\
\hline 12 & 1,010499 & $\mathrm{C}^{* *}$ & 0,0026 & $\mathrm{C}^{* *}$ & 0,105775 & $\mathrm{NC}$ & 0.03215 & $\mathrm{C}^{* *}$ & 0.0105 & $\mathrm{C}^{* *}$ \\
\hline 13 & & $\mathrm{NC}$ & & $\mathrm{C}^{* *}$ & 0,02 & $\mathrm{C}^{* * *}$ & & $\mathrm{C}^{* * *}$ & & $\mathrm{NC}$ \\
\hline 14 & 1,754717 & $\mathrm{NC}$ & 0,02668 & $\mathrm{C}^{* *}$ & 0,006004 & $\mathrm{C}^{* * *}$ & 0.02706 & $\mathrm{C}^{* * *}$ & 0.74468 & $\mathrm{NC}$ \\
\hline 15 & 0,436059 & $\mathrm{NC}$ & 0,043716 & $\mathrm{C}^{* *}$ & 0,494312 & $\mathrm{NC}$ & 0.010674 & $\mathrm{C}^{* *}$ & 0.573171 & $\mathrm{NC}$ \\
\hline 16 & 0,928365 & $\mathrm{NC}$ & 0,021006 & $\mathrm{C}^{* *}$ & 0,063654 & $\mathrm{NC}$ & 0.0025 & $\mathrm{C}^{* *}$ & 0.069 & $\mathrm{NC}$ \\
\hline 17 & 1,049062 & $\mathrm{C}^{* *}$ & 0,00822 & $\mathrm{C}^{* *}$ & 0,000677 & $\mathrm{C}^{* * *}$ & 0.00834 & $\mathrm{C}^{* *}$ & 0.05359 & $\mathrm{NC}$ \\
\hline 18 & 0,835366 & $\mathrm{NC}$ & 0,006167 & $\mathrm{C}^{* *}$ & 0,025697 & $\mathrm{C}^{* * *}$ & 0.005342 & $\mathrm{C}^{* *}$ & 0.20253 & $\overline{\mathrm{NC}}$ \\
\hline 19 & 2,734305 & $\mathrm{NC}$ & 0,16778 & $\mathrm{NC}$ & 0,250529 & $\mathrm{NC}$ & 0.2562 & $\mathrm{NC}$ & 1.7343 & $\mathrm{NC}$ \\
\hline 20 & 1,074319 & $\mathrm{NC}$ & 0,0165 & $\mathrm{C}^{* *}$ & 0,03738 & $\mathrm{C}^{* *}$ & 0.00791 & $\mathrm{C}^{* *}$ & 0.06977 & $\mathrm{NC}$ \\
\hline 21 & 1,10 & $\mathrm{NC}$ & 0,02474 & $\mathrm{C}^{* *}$ & 0,05219 & $\mathrm{NC}$ & 0.01167 & $\mathrm{C}^{* * *}$ & 0.098901 & $\mathrm{NC}$ \\
\hline 22 & 1,115615 & $\mathrm{NC}$ & 0,02452 & $\mathrm{C}^{* *}$ & 0,04137 & $\mathrm{C}^{* * *}$ & 0.01512 & $\mathrm{C}^{* *}$ & 0.1129 & $\mathrm{NC}$ \\
\hline 23 & 1,606667 & $\mathrm{NC}$ & 0,04822 & $\mathrm{C}^{* * *}$ & 0,01325 & $\mathrm{C}^{* * *}$ & 0.04655 & $\mathrm{C}^{* *}$ & 0.5847 & $\mathrm{NC}$ \\
\hline 24 & 0,98532 & $\mathrm{C}^{* *}$ & 0,001437 & $\mathrm{C}^{* *}$ & 0,06641 & $\mathrm{NC}$ & 0.007441 & $\mathrm{C}^{* *}$ & 0.015 & $\mathrm{C}^{* *}$ \\
\hline 25 & 1,188119 & $\mathrm{NC}$ & 0,02295 & $\mathrm{C}^{* *}$ & 0,05072 & $\mathrm{NC}$ & 0.01595 & $\mathrm{C}^{* *}$ & 0.18447 & $\mathrm{NC}$ \\
\hline 26 & 1,12867 & $\mathrm{NC}$ & 0,03967 & $\mathrm{C}^{* *}$ & 0,01192 & $\mathrm{C}^{* *}$ & 0.03557 & $\mathrm{C}^{* *}$ & 0.14734 & $\mathrm{NC}$ \\
\hline 27 & 0,455844 & $\mathrm{NC}$ & 0,080176 & $\mathrm{NC}$ & 0,022579 & $\mathrm{C}^{* *}$ & 0.078624 & $\mathrm{NC}$ & 0.546154 & $\mathrm{NC}$ \\
\hline 28 & 0,780718 & $\mathrm{NC}$ & 0,023909 & $\mathrm{C}^{* *}$ & 0,012923 & $\mathrm{C}^{* * *}$ & 0.022794 & $\mathrm{C}^{* * *}$ & 0.233333 & $\mathrm{NC}$ \\
\hline 29 & 2,057851 & $\mathrm{NC}$ & 0,0229 & $\mathrm{C}^{* *}$ & 0,205243 & $\mathrm{NC}$ & 0.03441 & $\mathrm{C}^{* * *}$ & 1.05556 & $\mathrm{NC}$ \\
\hline 30 & 1,176077 & $\mathrm{NC}$ & 0,0399 & $\mathrm{C}^{* * *}$ & 0,02479 & $\mathrm{C}^{* * *}$ & 0.03345 & $\mathrm{C}^{* *}$ & 0.17608 & $\mathrm{NC}$ \\
\hline
\end{tabular}




\section{Annexe 4 : Analyse des principaux reclassements et retraitements pour la transition d'un compte de résultat français à un compte de résultat IAS/IFRS}

\begin{tabular}{|c|c|c|c|c|c|c|c|c|c|c|c|c|c|c|c|c|c|c|c|c|c|c|}
\hline \multirow[t]{2}{*}{ Entreprises } & \multicolumn{2}{|l|}{1} & \multicolumn{2}{|l|}{2} & \multicolumn{2}{|l|}{3} & \multicolumn{2}{|l|}{4} & \multicolumn{2}{|l|}{5} & \multicolumn{2}{|l|}{8} & \multicolumn{2}{|l|}{9} & \multicolumn{2}{|l|}{10} & \multicolumn{2}{|l|}{11} & \multicolumn{2}{|l|}{13} & \multicolumn{2}{|l|}{15} \\
\hline & Rc & $\mathrm{Rt}$ & $\mathrm{Rc}$ & $\mathrm{Rt}$ & Rc & Rt & Rc & $\mathrm{Rt}$ & Rc & $\mathrm{Rt}$ & $\mathrm{Rc}$ & $\mathrm{Rt}$ & $\mathrm{Rc}$ & $\mathrm{Rt}$ & $\mathrm{Rc}$ & $\mathrm{Rt}$ & $\mathrm{Rc}$ & $\mathrm{Rt}$ & Rc & $\mathrm{Rt}$ & Rc & $\mathrm{Rt}$ \\
\hline $\begin{array}{l}\text { Ventes de } \\
\text { marchandises }\end{array}$ & & & & & & & $\mathrm{X}$ & & & & & & & & & & & & & & & \\
\hline $\begin{array}{l}\text { Production } \\
\text { vendue }\end{array}$ & & & & & & & & & & & & & & & & & & & & & & \\
\hline $\begin{array}{l}\text { Chiffre } \\
\text { d'affaires }\end{array}$ & & & $\mathbf{X}$ & & & & & & & & & $\mathrm{X}$ & $\mathrm{X}$ & & & & & & $\mathrm{X}$ & & & $\mathrm{X}$ \\
\hline $\begin{array}{l}\text { Production } \\
\text { stockée et } \\
\text { immobilisée }\end{array}$ & & & $\mathrm{X}$ & & & & $\mathrm{X}$ & & & & & & & & & & & & & & & \\
\hline $\begin{array}{l}\text { Autres produits } \\
\text { d'exploitation }\end{array}$ & & & $\mathrm{X}$ & $\mathrm{X}$ & $\mathrm{X}$ & & $\mathrm{X}$ & $\mathrm{X}$ & $\mathrm{X}$ & $\mathrm{X}$ & & $\mathrm{X}$ & $\mathrm{X}$ & & $\mathrm{X}$ & $\mathrm{X}$ & & & $\mathrm{X}$ & & & $\mathrm{X}$ \\
\hline $\begin{array}{l}\text { Total des } \\
\text { produits } \\
\text { d'exploitation }\end{array}$ & & & & & & & & & & & & & & & & & & & & & & \\
\hline $\begin{array}{l}\text { Achats de } \\
\text { marchandises }\end{array}$ & & & & & & & $\mathrm{X}$ & & & $\mathrm{X}$ & & & & & & $\mathrm{X}$ & & & $\mathrm{X}$ & & & \\
\hline $\begin{array}{l}\text { Variation de } \\
\text { stocks }\end{array}$ & & & & & & & & & & & & & & & & & & & & & & \\
\hline $\begin{array}{l}\text { Achats de } \\
\text { matières } \\
\text { premières }\end{array}$ & & & & & & & & & & & & & & & & & & & & & & \\
\hline $\begin{array}{l}\text { Autres achats et } \\
\text { charges } \\
\text { externes }\end{array}$ & & $\mathrm{X}$ & & & & & & & & & & & $\mathrm{X}$ & $\mathrm{X}$ & & $\mathrm{X}$ & & & $\mathrm{X}$ & $\mathrm{X}$ & & $\mathrm{X}$ \\
\hline Impôts et taxes & & & & & & & $\mathrm{X}$ & & & & & & $\mathrm{X}$ & $\mathrm{X}$ & & & & & $\mathrm{X}$ & & & \\
\hline $\begin{array}{l}\text { Charges du } \\
\text { personnel }\end{array}$ & & $\mathrm{X}$ & & & & & $\mathrm{X}$ & $\mathrm{X}$ & & $\mathrm{X}$ & $\mathrm{X}$ & $\mathrm{X}$ & $\mathrm{X}$ & $\mathrm{X}$ & & & & $\mathrm{X}$ & $\mathrm{X}$ & $\mathrm{X}$ & & $\mathrm{X}$ \\
\hline $\begin{array}{l}\text { Amortissements } \\
\text { et provisions }\end{array}$ & & $\mathrm{X}$ & & & $\mathrm{X}$ & $\mathrm{X}$ & $\mathrm{X}$ & $\mathrm{X}$ & & $\mathrm{X}$ & & & $\mathrm{X}$ & $\mathrm{X}$ & & $\mathrm{X}$ & & $\mathrm{X}$ & $\mathrm{X}$ & $\mathrm{X}$ & & $\mathrm{X}$ \\
\hline $\begin{array}{l}\text { Autres charges } \\
\text { d'exploitation }\end{array}$ & & & & & & & $\mathrm{X}$ & & & & $\mathrm{X}$ & & $\mathrm{X}$ & $\mathrm{X}$ & $\mathrm{X}$ & $\mathrm{X}$ & & $\mathrm{X}$ & $\mathrm{X}$ & $\mathrm{X}$ & & $\mathrm{X}$ \\
\hline $\begin{array}{l}\text { Total des } \\
\text { charges } \\
\text { d'exploitation } \\
\end{array}$ & & & & & & & & & & & & & & & & & & & & & & \\
\hline $\begin{array}{l}\text { Produits } \\
\text { financiers }\end{array}$ & & $\mathrm{X}$ & $\mathrm{X}$ & $\mathrm{X}$ & & & $\mathrm{X}$ & & $\mathrm{X}$ & & $\mathrm{X}$ & & $\mathrm{X}$ & $\mathrm{X}$ & & $\mathrm{X}$ & & & & & & \\
\hline $\begin{array}{l}\text { Charges } \\
\text { financières }\end{array}$ & & $\mathrm{X}$ & & & & $\mathrm{X}$ & $\mathrm{X}$ & & $\mathrm{X}$ & & $\mathrm{X}$ & & $\mathrm{X}$ & & & $\mathrm{X}$ & & $\mathrm{X}$ & $\mathrm{X}$ & $\mathrm{X}$ & & \\
\hline $\begin{array}{l}\text { Résultat } \\
\text { financier }\end{array}$ & & & & & & & & & & & & & & & & $\mathrm{X}$ & & & $\mathrm{X}$ & & & $\mathrm{X}$ \\
\hline $\begin{array}{l}\text { Résultat } \\
\text { courant }\end{array}$ & & & & & & & & & & & & & & & & & & & & & & \\
\hline $\begin{array}{l}\text { Résultat } \\
\text { exceptionnel }\end{array}$ & & & & & $\mathrm{X}$ & & & & & & $\mathrm{X}$ & & $\mathrm{X}$ & $\mathrm{X}$ & $\mathrm{X}$ & & & $\mathrm{X}$ & $\mathrm{X}$ & & & \\
\hline $\begin{array}{l}\text { Impôt sur les } \\
\text { bénéfices }\end{array}$ & & & & $\mathrm{X}$ & & $X$ & $\mathrm{X}$ & $\mathrm{X}$ & & $\mathrm{X}$ & & & $\mathrm{X}$ & $\mathrm{X}$ & & $\mathrm{X}$ & & $\mathrm{X}$ & $\mathrm{X}$ & $\mathrm{X}$ & & $\mathrm{X}$ \\
\hline $\begin{array}{l}\text { Résultat des } \\
\text { sociétés } \\
\text { intégrées } \\
\end{array}$ & & & & & & & & $\mathbf{X}$ & & & & & & & & & & & & & & \\
\hline $\begin{array}{l}\text { Quote-part des } \\
\text { sociétés mises } \\
\text { en équi }\end{array}$ & & & $\mathrm{X}$ & & & & $\mathrm{X}$ & & & & $\mathrm{X}$ & & & & & & & $\mathrm{X}$ & $\mathrm{X}$ & $\mathrm{X}$ & & $\mathrm{X}$ \\
\hline $\begin{array}{l}\text { Amortissement } \\
\text { écart } \\
\text { acquisition }\end{array}$ & & & & & & & $\mathrm{X}$ & $\mathrm{X}$ & & $\mathrm{X}$ & $\mathrm{X}$ & $\mathrm{X}$ & $\mathrm{X}$ & $\mathrm{X}$ & & $\mathrm{X}$ & & $\mathrm{X}$ & $\mathrm{X}$ & $\mathrm{X}$ & & \\
\hline $\begin{array}{l}\text { Résultat net de } \\
\text { l'ensemble } \\
\text { consolidé }\end{array}$ & & & & & & & & & & & & & & & & & & & & & & \\
\hline $\begin{array}{l}\text { Intérêts } \\
\text { minoritaires }\end{array}$ & & & & $\mathrm{X}$ & & & $\mathrm{X}$ & & & & & & $X$ & & & $\mathrm{X}$ & & $\mathrm{X}$ & & & & $\mathrm{X}$ \\
\hline $\begin{array}{l}\text { Résultat net } \\
\text { part du groupe }\end{array}$ & & & & & & & & & & & & & & & & & & & & & & \\
\hline
\end{tabular}




\begin{tabular}{|c|c|c|c|c|c|c|c|c|c|c|c|c|c|c|c|c|c|c|c|c|c|c|}
\hline \multirow[t]{2}{*}{ Entreprises } & \multicolumn{2}{|l|}{16} & \multicolumn{2}{|l|}{17} & \multicolumn{2}{|l|}{18} & \multicolumn{2}{|l|}{19} & \multicolumn{2}{|l|}{20} & \multicolumn{2}{|l|}{21} & \multicolumn{2}{|l|}{22} & \multicolumn{2}{|l|}{26} & \multicolumn{2}{|l|}{27} & \multicolumn{2}{|l|}{28} & \multicolumn{2}{|l|}{29} \\
\hline & $\mathrm{Rc}$ & $\mathrm{Rt}$ & $\mathrm{Rc}$ & $\mathrm{Rt}$ & Rc & $\mathrm{Rt}$ & Rc & $\mathrm{Rt}$ & Rc & $\mathrm{Rt}$ & $\mathrm{Rc}$ & $\mathrm{Rt}$ & $\mathrm{Rc}$ & $\mathrm{Rt}$ & $\mathrm{Rc}$ & $\mathrm{Rt}$ & $\mathrm{Rc}$ & $\mathrm{Rt}$ & $\mathrm{Rc}$ & $\mathrm{Rt}$ & $\mathrm{Rc}$ & $\mathrm{Rt}$ \\
\hline $\begin{array}{l}\text { Ventes de } \\
\text { marchandises }\end{array}$ & & & & & & & & & & & & & & & & & & & & & & \\
\hline $\begin{array}{l}\text { Production } \\
\text { vendue }\end{array}$ & & & & & & & & & & & & & & & & & & & & & & \\
\hline $\begin{array}{l}\text { Chiffre } \\
\text { d'affaires }\end{array}$ & & $\mathrm{X}$ & $\mathrm{X}$ & & & & & $\mathrm{X}$ & & & $\mathrm{X}$ & & $\mathrm{X}$ & & & $\mathrm{X}$ & & & & & & $\mathrm{X}$ \\
\hline $\begin{array}{l}\text { Production } \\
\text { stockée et } \\
\text { immobilisée }\end{array}$ & & & & & & & & & & & & & & & & & & & & & & \\
\hline $\begin{array}{l}\text { Autres produits } \\
\text { d'exploitation }\end{array}$ & & $\mathrm{X}$ & & & & $\mathrm{X}$ & $\mathrm{X}$ & & & & $\mathrm{X}$ & $\mathrm{X}$ & $\mathrm{X}$ & & & & & $\mathrm{X}$ & & & & $\mathrm{X}$ \\
\hline $\begin{array}{l}\text { Total des } \\
\text { produits } \\
\text { d'exploitation }\end{array}$ & & & & & & & & & & & & & & & & & & & & & & \\
\hline $\begin{array}{l}\text { Achats de } \\
\text { marchandises }\end{array}$ & & & & & & & $\mathrm{X}$ & $\mathrm{X}$ & & & & & & & & $\mathrm{X}$ & & & & $\mathrm{X}$ & & \\
\hline $\begin{array}{l}\text { Variation de } \\
\text { stocks }\end{array}$ & & & & & & & & & & & & & & & & & & & & & & \\
\hline $\begin{array}{l}\text { Achats de } \\
\text { matières } \\
\text { premières }\end{array}$ & & & & & & & & & & & & & & & & & & & & & & \\
\hline $\begin{array}{l}\text { Autres achats et } \\
\text { charges } \\
\text { externes }\end{array}$ & & $\mathrm{X}$ & & & & $\mathrm{X}$ & $\mathrm{X}$ & $\mathrm{X}$ & & & $\mathrm{X}$ & & $\mathrm{X}$ & $\mathrm{X}$ & & & & & & & & \\
\hline Impôts et taxes & & $\mathrm{X}$ & & & & & & & & & $\mathrm{X}$ & & & & & & & & & & & \\
\hline $\begin{array}{l}\text { Charges du } \\
\text { personnel }\end{array}$ & & $\mathrm{X}$ & & $\mathrm{X}$ & & $\mathrm{X}$ & & & & $\mathrm{X}$ & $\mathrm{X}$ & $\mathrm{X}$ & & & & & & $\mathrm{X}$ & & & & \\
\hline $\begin{array}{l}\text { Amortissements } \\
\text { et provisions }\end{array}$ & & $\mathrm{X}$ & & $\mathrm{X}$ & & $\mathrm{X}$ & & $\mathrm{X}$ & & $\mathrm{X}$ & $\mathrm{X}$ & $\mathrm{X}$ & $\mathrm{X}$ & $\mathrm{X}$ & & $\mathrm{X}$ & & $\mathrm{X}$ & & & & $\mathrm{X}$ \\
\hline $\begin{array}{l}\text { Autres charges } \\
\text { d'exploitation }\end{array}$ & & $\mathrm{X}$ & & $\mathrm{X}$ & & $\mathrm{X}$ & $\mathrm{X}$ & $\mathrm{X}$ & & $\mathrm{X}$ & $\mathrm{X}$ & & $\mathrm{X}$ & & & $\mathrm{X}$ & & $\mathrm{X}$ & & $\mathrm{X}$ & & $\mathrm{X}$ \\
\hline $\begin{array}{l}\text { Total des } \\
\text { charges } \\
\text { d'exploitation }\end{array}$ & & & & & & & & & & & & & & & & & & & & & & \\
\hline $\begin{array}{l}\text { Produits } \\
\text { financiers }\end{array}$ & & & & $\mathrm{X}$ & & $\mathrm{X}$ & $\mathrm{X}$ & & & & & & & & & $\mathrm{X}$ & & & & & & \\
\hline $\begin{array}{l}\text { Charges } \\
\text { financières }\end{array}$ & & & & $\mathrm{X}$ & & $\mathrm{X}$ & $\mathrm{X}$ & $\mathrm{X}$ & & & & & & & & & & & & $\mathrm{X}$ & & \\
\hline $\begin{array}{l}\text { Résultat } \\
\text { financier }\end{array}$ & & $\mathrm{X}$ & & & & & & & & & $\mathrm{X}$ & $\mathrm{X}$ & & & & & & & & & & $\mathrm{X}$ \\
\hline $\begin{array}{l}\text { Résultat } \\
\text { courant }\end{array}$ & & & & & & & & & & & & & & & & & & & & & & \\
\hline $\begin{array}{l}\text { Résultat } \\
\text { exceptionnel }\end{array}$ & & & $\mathrm{X}$ & $\mathrm{X}$ & & & $\mathrm{X}$ & $\mathrm{X}$ & & & $\mathrm{X}$ & & & & & & & & & & & $\mathrm{X}$ \\
\hline $\begin{array}{l}\text { Impôt sur les } \\
\text { bénéfices }\end{array}$ & & $\mathrm{X}$ & & $\mathrm{X}$ & & & $\mathrm{X}$ & $\mathrm{X}$ & & $\mathrm{X}$ & & $\mathrm{X}$ & $\mathrm{X}$ & $\mathrm{X}$ & & $\mathrm{X}$ & & & & & & $\mathrm{X}$ \\
\hline $\begin{array}{l}\text { Résultat des } \\
\text { sociétés } \\
\text { intégrées }\end{array}$ & & & & & & & & & & & & & & & & & & & & & & \\
\hline $\begin{array}{l}\text { Quote-part des } \\
\text { sociétés mises } \\
\text { en équi }\end{array}$ & & $\mathrm{X}$ & & & & & $\mathrm{X}$ & $\mathrm{X}$ & & $\mathrm{X}$ & & & & & & $\mathrm{X}$ & & & & & & \\
\hline $\begin{array}{l}\text { Amortissement } \\
\text { écart } \\
\text { acquisition }\end{array}$ & & $\mathrm{X}$ & & $\mathrm{X}$ & & & & & & $\mathrm{X}$ & $\mathrm{X}$ & & $\mathrm{X}$ & $\mathrm{X}$ & & $\mathrm{X}$ & & $\mathrm{X}$ & & $\mathrm{X}$ & & \\
\hline $\begin{array}{l}\text { Résultat net de } \\
\text { l'ensemble } \\
\text { consolidé }\end{array}$ & & & & & & & & & & & & & & & & & & & & & & \\
\hline $\begin{array}{l}\text { Intérêts } \\
\text { minoritaires }\end{array}$ & & $\mathrm{X}$ & & $\mathrm{X}$ & & & $\mathrm{X}$ & $\mathrm{X}$ & & & & $\mathrm{X}$ & & & & $\mathrm{X}$ & & & & & & $\mathrm{X}$ \\
\hline $\begin{array}{l}\text { Résultat net } \\
\text { part du groupe }\end{array}$ & & & & & & & & & & & & & & & & & & & & & & \\
\hline
\end{tabular}


Annexe 5: Résultats des régressions

\begin{tabular}{|l|l|c|c|c|c|c|}
\hline Variables & & $\mathrm{COMPA}$ & $\mathrm{VARR}_{\mathrm{RN}}$ & $\mathrm{VAR}_{\mathrm{AN}}$ & $\mathrm{VAR}_{\mathrm{RCP}}$ & $\mathrm{VAR}_{\mathrm{BA}}$ \\
\hline \multirow{3}{*}{$\mathrm{CA}$} & Sens & + & + & + & + & + \\
\cline { 2 - 7 } & Prob & $0.008^{*} *$ & $0.069 *$ & 0.15 & $0.08 *$ & 0.19 \\
\hline \multirow{2}{*}{ SECT } & Sens & + & + & + & + & + \\
\cline { 2 - 7 } & Prob & 0.157 & 0.777 & 0.98 & 0.22 & 0.64 \\
\hline \multicolumn{7}{|c}{} \\
\end{tabular}

Tableau des corrélations

\begin{tabular}{|c|c|c|c|c|c|c|c|}
\hline & CA & SECT & COMPA & VARR $_{\text {RN }}$ & VARR $_{A N}$ & VARR $_{\text {RCP }}$ & VARR $_{\text {BA }}$ \\
\hline CA & 1 & 0.085 & -0.119 & -0.085 & 0.068 & -0.148 & -0.084 \\
\hline SECT & 0.085 & 1 & 0.196 & -0.111 & 0.311 & 0.149 & -0.111 \\
\hline COMPA & -0.119 & 0.196 & 1 & 0.196 & -0.222 & -0.087 & 0.522 \\
\hline VARR $_{\text {RN }}$ & -0.085 & -0.111 & 0.196 & 1 & 0.200 & 0.670 & 0.166 \\
\hline VARR $_{\text {AN }}$ & 0.068 & 0.311 & -0.222 & 0.200 & 1 & 0.298 & -0.133 \\
\hline VARR $_{\text {RP }}$ & -0.148 & 0.149 & -0.087 & 0.670 & 0.298 & 1 & 0.149 \\
\hline VARR $_{\text {BA }}$ & -0.084 & -0.111 & 0.522 & 0.166 & -0.133 & 0.149 & 1 \\
\hline
\end{tabular}

